\title{
FABRICATION AND CHARACTERIZATION OF BIOMAGNETIC COMPOSITE MATERIAL
}

\author{
Yekbun AVŞAR TEYMUR ${ }^{1}$, Fuat GÜZEL ${ }^{* 2}$, Gülbahar AKKAYA SAYĞILI ${ }^{2}$ \\ ${ }^{1}$ Department of Chemistry, Institute of Natural and Applied Sciences, Dicle University, 21280 Diyarbakır, Turkey \\ ${ }^{2 *}$ Department of Chemistry, Faculty of Education, Dicle University, 21280 Diyarbakır, Turkey
}

\begin{abstract}
In this study, the synthesis and characterization of a biomagnetic composite material was achieved by a simple and cost effective method. Tomato processing waste was successfully converted into a magnetic material via embedding $\mathrm{Fe}_{3} \mathrm{O}_{4}$ nanoparticles to its structure. Due to its low cost and ease of application, co-precipitation method was used for loading the magnetite nanoparticles. Characterization studies were carried out with Fourier transform infrared spectroscopy, scanning electron microscopy and vibrating sample magnetometer spectroscopy and the outcomes of the analyses of non-magnetic and magnetic material were compared.
\end{abstract}

Key words: tomato processing waste, magnetic composite, magnetite nanoparticles, characterization

\section{Introduction}

Interest in nanotechnologies and nanoscale materials, particularly magnetic nanoparticles (MNPs), has grown recently and their applications have attracted the attention of both the research and industrial communities in the chemical, environmental and medical sectors. Their effective application in membrane separation water treatment and purification processes has been demonstrated [1]. Although there are many pure phases of iron oxide in nature, the most popular MNPs are the nanoscale zero-valent iron (nZVI), $\mathrm{Fe}_{3} \mathrm{O}_{4}$ and $\gamma-\mathrm{Fe}_{2} \mathrm{O}_{3}$. They possess different physicochemical properties originating from the difference in their iron oxidation states and their capability for contaminant removal. Among them, magnetite $\left(\mathrm{Fe}_{3} \mathrm{O}_{4}\right)$, which is a ferromagnetic black color iron oxide of both $\mathrm{Fe}(\mathrm{II})$ and $\mathrm{Fe}(\mathrm{III})$, has been the most extensively studied. Magnetite is the preferred type because of the presence of the $\mathrm{Fe}^{2+}$ state with the potential of acting as an electron donor. Fabrication and synthesis techniques of magnetic nanoparticles have been reviewed by many authors such as co-precipitation method [2], aerosol route [3], hydrothermal reaction [4], oxidative precipitation [5], organic precursor method [6], sonochemical decomposition [7], and sol-gel synthesis technique [8]. In this study, coprecipitation method was used to convert tomato processing waste a magnetic composite material. The synthesized material was characterized and the properties of the composite were compared with non-magnetic material.

\section{Materials and Method}

\subsection{Preparation of the biosorbent}

Tomato processing waste was supplied from a fruit-vegetable processing mill at Adana, Turkey.

*Corresponding author: Address: Department of Chemistry, Faculty of Education, Dicle University, 21280 Diyarbakır, Turkey. E-mail address: fguzel@dicle.edu.tr, Phone: +904122481000 
Tomato processing waste was washed with hot distilled for several times and then dried in sunlight. The dried tomato processing wastewas sized to 14 mesh particle size and then washed with ultra-pure water again. It was dried at $70{ }^{\circ} \mathrm{C}$ in an oven for $24 \mathrm{~h}$.

\subsection{Preparation of the biomagnetic composite}

In order to synthesize biomagnetic composite adsorbent, co-precipitation method was used as follows: The stoichiometric amounts of $\mathrm{FeCl}_{3}$ and $\mathrm{FeCl}_{2}$ were dissolved in distilled water. A certain amount of tomato processing waste was added to the mixture under vigorous stirring. Then $\mathrm{NaOH}$ solution was used to adjust the $\mathrm{pH}$ of the solution to the range of 10-11. After $\mathrm{pH}$ adjustment, the solution was kept at $100{ }^{\circ} \mathrm{C}$ for $4 \mathrm{~h}$. Then, the solution was filtrated, washed with distilled water several times and dried at $105{ }^{\circ} \mathrm{C}$ for $24 \mathrm{~h}$. The synthesized adsorbent was labelled as magnetic tomato processing waste and stored in glass bottles.

\subsection{Characterization studies}

The surface chemical functionalities of samples was qualitatively determined by Attenuated total reflection Fourier transform infrared (ATR-FTIR) spectroscopy with a Perkin Elmer spectrum 100 spectrometer between $4000-400 \mathrm{~cm}^{-1}$.

The surface morphology was identified by the scanning electron microscopy (SEM) instrument (Carl Zeiss Ultra Plus, UK). Micrographs of samples were observed by the assistance of coated with platinum, operating at $10 \mathrm{kV}$.

Vibrating sample magnetometer (VSM 7404, Lake Shore Cryotronics, USA) was utilized to magnetic features of the synthesized material.

\section{Results and Discussion}

\subsection{FTIR analysis}

The spectra of raw tomato waste and magnetic tomato waste were compared in Fig.1. The peak at $3286 \mathrm{~cm}^{-1}$ is due to O-H vibration. The absorption bands located around 2923 and $2855 \mathrm{~cm}^{-1} \mathrm{can}$ be assigned to the aliphatic $\mathrm{C}-\mathrm{H}$ vibrations. Two peaks at 1740 and $1632 \mathrm{~cm}^{-1}$ assigned to the $\mathrm{C}=\mathrm{O}$ group and indicates the existence of aldehyde groups. The signals at 1443 and $1157 \mathrm{~cm}^{-1}$ are due to the $\mathrm{C}-\mathrm{C}$ bindings. The peak at $1029 \mathrm{~cm}-1$ indicates the stretching vibration of $\mathrm{C}-\mathrm{O}$ bond. When the spectra of tomato processing waste and magnetic tomato processing waste were compared, it can be observed from the figure that, the peak intensities have changed after magnetite nanoparticles loading. In the spectra of magnetic composite, a new peak was occurred at $556 \mathrm{~cm}^{-1}$ that confirms the successful loading of the magnetite nanoparticles to the structure of tomato waste. 


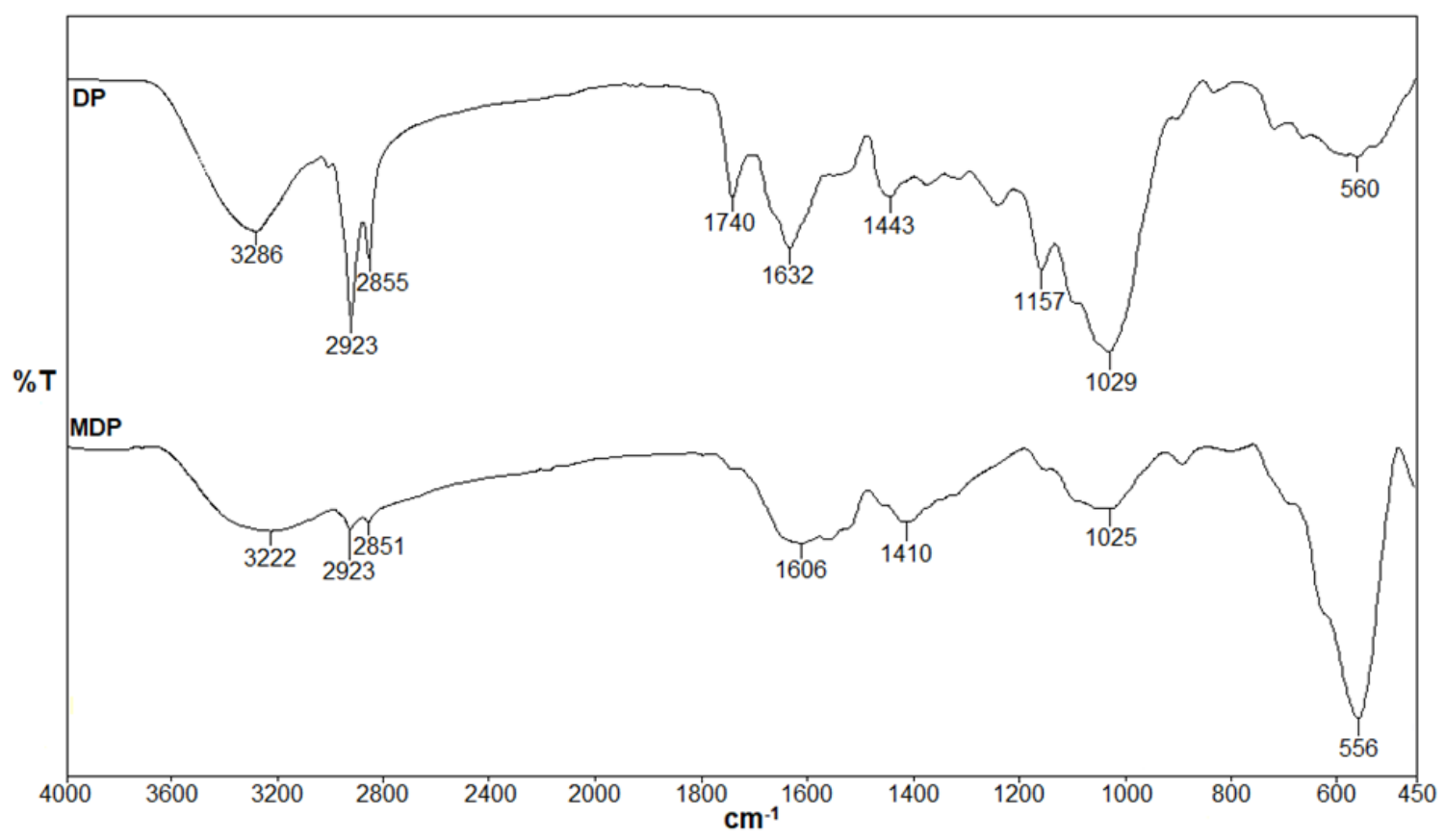

Figure 1. FTIR spectrum of tomato processing waste and its magnetic composite.

\subsection{SEM analysis}

SEM micrographs of tomato processing waste and magnetic tomato processing waste are shown in Fig.2. It can be seen from the figure that, the surface of tomato processing waste and magnetic tomato processing waste are different from each other. The surface of magnetic tomato processing waste is has an unregular structure and has some cavities and cracks.
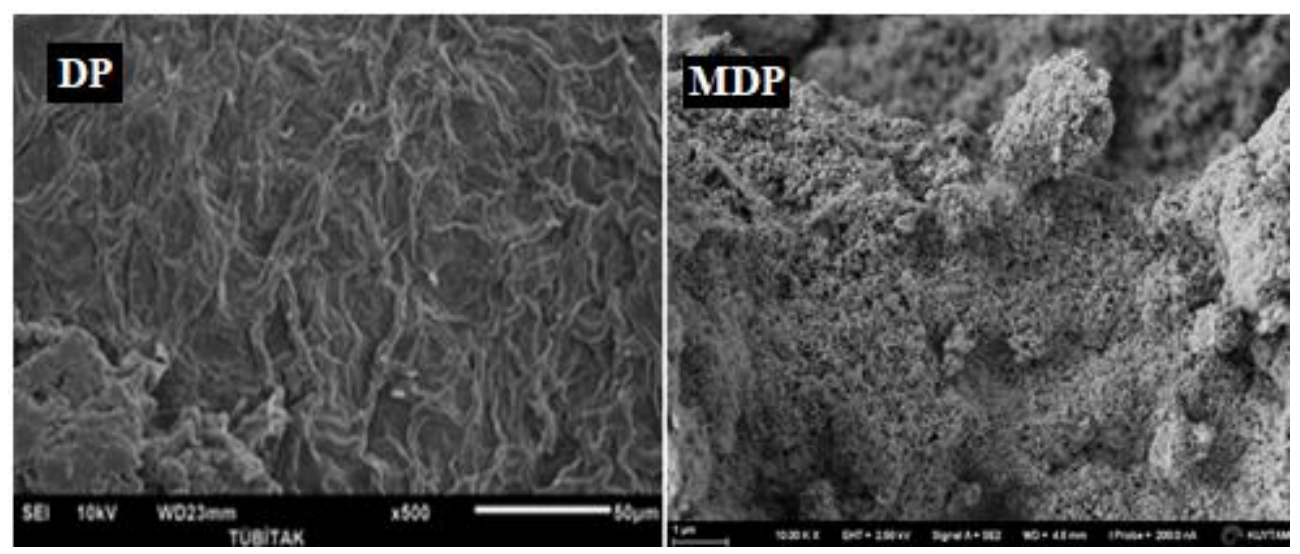

Figure 2. SEM images of tomato processing waste and its magnetic composite.

\subsection{VSM analysis}

VSM curves of the magnetic tomato processing waste is seen in Figure 3 and indicates the 
magnetic property of the synthesized material. Also the inset figure shows that the synthesized composite material is magnetically separable.

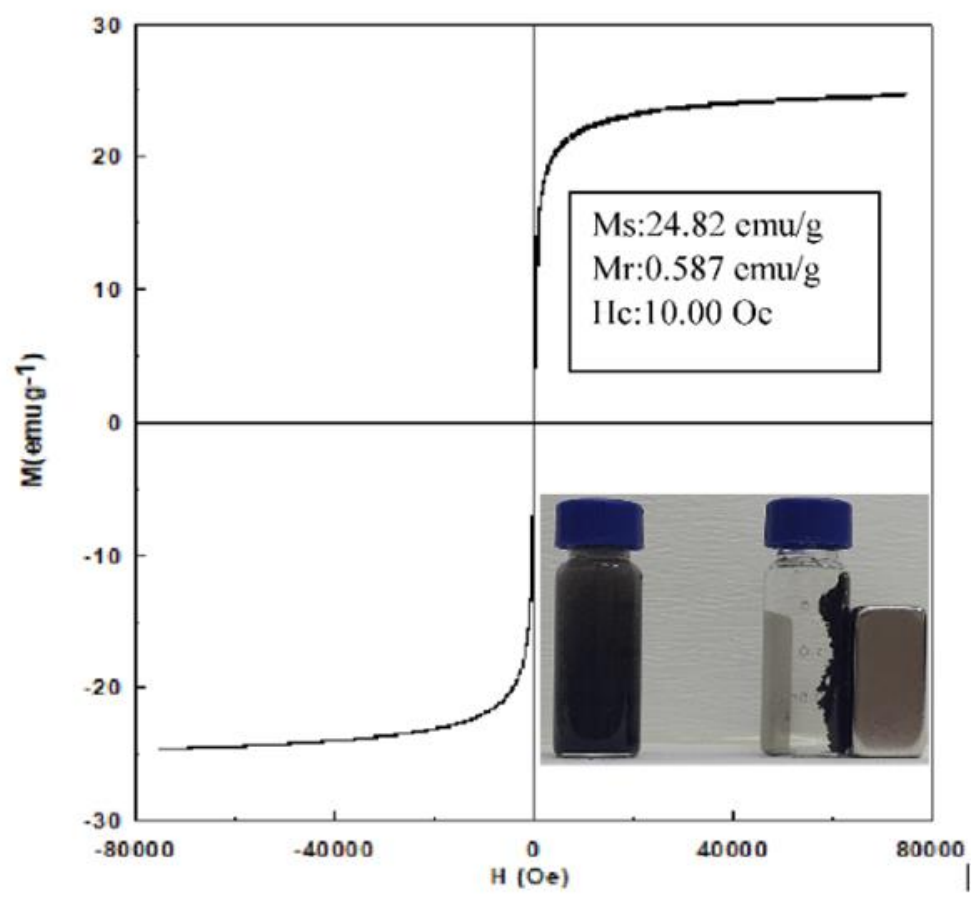

Figure 3. VSM curves of MTPW composite.

\section{Conclusions}

A novel magnetically separable composite material was synthesized from a global waste. The fabricated material was characterized in terms of surface structure and magnetic features and also compared with its non-magnetic form. This study indicated that, tomato waste could be used as a promising feedstock for production of magnetic materials due to its abundantly avalability and low cost.

\section{Acknowledgement}

The authors thank to the Scientific Research Projects Unit of Dicle University (project code: ZGEF-17-024) for the financial support.

\section{References}

[1] Farmany, A., Mortazavi, S. S., Mahdavi, H., "Ultrasound-assisted synthesis of $\mathrm{Fe}_{3} \mathrm{O}_{4} / \mathrm{SiO}_{2}$ core/shell with enhanced adsorption capacity for diazinon removal", Journal of Magnetism and Magnetic Materials, Vol.416, pp.75-80, 2016.

[2] Petcharoen, K., Sirivat, A., "Synthesis and characterization of magnetite nanoparticles via the chemical co-precipitation method", Material Science Engineering B Solid State 
Materials Advanced Technologies, Vol.177, pp.421-427, 2012.

[3] Singhal, S., Chandra, K., "Cation distribution and magnetic properties in chromiumsubstituted nickel ferrites prepared using aerosol route", Journal of Solid State Chemistry, Vol.180, pp.296-300, 2007.

[4] Ozel, F., Kockar, H., "Growth and characterizations of magnetic nanoparticles under hydrothermal conditions: Reaction time and temperature", Journal of Magnetism and Magnetic Materials, Vol.373, pp.213-216, 2015.

[5] Wan, C., Li, J., "Synthesis of well-dispersed magnetic $\mathrm{CoFe}_{2} \mathrm{O}_{4}$ nanoparticles in cellulose aerogels via a facile oxidative co-precipitation method", Carbohydrate Polymers, Vol.134, pp.144-150, 2015.

[6] Mohamed, R.M., Rashad, M.M., Haraz, F.A., Sigmund, W., "Structure and magnetic properties of nanocrystalline cobalt ferrite powders synthesized using organic acid precursor method", Journal of Magnetism and Magnetic Materials, Vol.322, pp.20582064, 2010.

[7] Aliramaji, S., Zamanian, A., Sohrabijam, Z., "Characterization and synthesis of magnetite nanoparticles by innovative sonochemical method”, Procedia Material Science, Vol.11, pp.265-269, 2015.

[8] Wang, X., Zhang, P., Gao, J., Chen, X., Yang, H., "Facile synthesis and magnetic properties of $\mathrm{Fe}_{3} \mathrm{C} / \mathrm{C}$ nanoparticles via a sol-gel process", Dyes and Pigments, Vol.112, pp.305-310, 2015. 